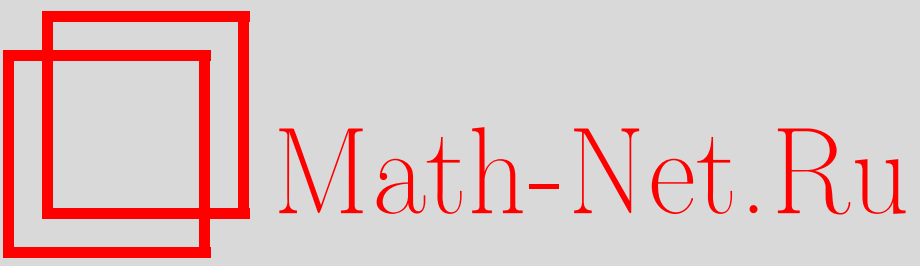

А. Е. Вязилов, Эмпирические процессы в $\operatorname{GARCH}(1,1)$-модели и робастное оценивание параметров, УМН, 2001, том 56, выпуск 5, 179-180

DOI: https://doi.org/10.4213/rm442

Использование Общероссийского математического портала Math-Net.Ru подразумевает, что вы прочитали и согласны с пользовательским соглашением

http: //www. mathnet.ru/rus/agreement

Параметры загрузки:

IP : 54.198 .67 .100

26 апреля 2023 г., 12:29:18 


\title{
ЭМПИРИЧЕСКИЕ ПРОЦЕССЫ В GАRСН(1,1)-МОДЕЛИ И РОБАСТНОЕ ОЦЕНИВАНИЕ ПАРАМЕТРОВ
}

\author{
А.Е. Вязилов
}

1. Модель GARCH(1,1) (Generalized AutoRegressive Conditional Heteroscedasticity) задается соотношениями

$$
y_{k}=\sigma_{k} \varepsilon_{k}, \quad \sigma_{k}^{2}=\alpha+\beta y_{k-1}^{2}+\gamma \sigma_{k-1}^{2}, \quad k \in \mathbb{Z},
$$

где $\left\{\varepsilon_{k}\right\}$ - независимые одинаково распределенные случайные величины с функцией распределения $G(x), \mathrm{E}_{1}^{2}=1 ; \mathbf{a}=(\alpha, \beta, \gamma)$ - вектор неизвестных параметров, $\alpha, \beta, \gamma>0, \beta+\gamma<1$. Тогда существует единственное строго стационарное и эргодическое решение $\left\{y_{k}\right\}$.

Одна из основных задач статистического анализа модели (1) - оценивание неизвестных параметров. Если функция $G(x)$ известна, несложно построить оценки максимального правдоподобия (ОМП) или им асимптотически эквивалентные, см. [1]. Более того, $n^{1 / 2}$-асимптотически нормальные оценки можно построить и в непараметрической ситуации, см. там же. Однако, описанные в литературе оценки неустойчивы к грубым выбросам.

В данной работе строится класс $n^{1 / 2}$-асимптотически нормальных оценок с конечной чувствительностью в схеме засорения данных одиночными выбросами (необходимые определения см. в [2]). Решение этой задачи нетривиально даже в параметрической ситуации, которую мы и рассмотрим. Наш подход основан на использовании остаточных эмпирических процессов и примыкает к [3], где строятся робастные непараметрические оценки в модели ARCH.

2. Пусть $y_{0}, \ldots, y_{n}$ - наблюдения из стационарного решения уравнений (1). Положим

$$
\sigma_{k}^{2}(\mathbf{t}):=t_{1}+t_{2} y_{k-1}^{2}+t_{3} \sigma_{k-1}^{2}(\mathbf{t}), \quad \varepsilon_{k}(\mathbf{t}):=\left\{\begin{array}{ll}
\frac{y_{k}}{\sigma_{k}(\mathbf{t})}, & \text { если } \sigma_{k}^{2}(\mathbf{t})>0, \\
1, & \text { если } \sigma_{k}^{2}(\mathbf{t}) \leqslant 0,
\end{array} \quad k=1, \ldots, n,\right.
$$

где $\mathbf{t}=\left(t_{1}, t_{2}, t_{3}\right) \in \mathbb{R}^{3}, \sigma_{0} \equiv 0$. Введем для $x \in \mathbb{R}$ остаточные әмпирические прочессы

$U_{n}(x, \mathbf{t}):=n^{-1 / 2} \sum_{k=1}^{n} f\left(\widehat{\mathbf{y}}_{k-1} ; \mathbf{t}\right) I\left\{\varepsilon_{k}(\mathbf{t}) \leqslant x\right\}, \quad U_{n}(x):=n^{-1 / 2} \sum_{k=1}^{n} f\left(\mathbf{y}_{k-1} ; \mathbf{a}\right) I\left\{\varepsilon_{k} \leqslant x\right\}$,

где $\mathbf{y}_{k}=\left(\ldots, y_{k-1}, y_{k}\right), \widehat{\mathbf{y}}_{k}=\left(\ldots, 0, y_{0}, \ldots, y_{k}\right)$. Нам потребуются условия:

(i) $G(x)$ - строго монотонна на множестве $\{x \in \mathbb{R}: 0<G(x)<1\}$;

(ii) существует дифференцируемая плотность $g(x)=G^{\prime}(x)$ и $\sup _{x \in \mathbb{R}} x^{2}\left|g^{\prime}(x)\right|<\infty$;

(iii) $\mathrm{E}\left|f\left(\mathbf{y}_{1} ; \mathbf{a}\right)\right|^{4}<\infty, n^{-1 / 2} \sum_{k=1}^{n}\left|f\left(\mathbf{y}_{k} ; \mathbf{a}\right)-f\left(\widehat{\mathbf{y}}_{k} ; \mathbf{a}\right)\right|=o_{p}(1)$;

(iv) существуют $\dot{f}^{i}=\partial f / \partial t_{i}$ и $\mathrm{E}\left\|\dot{f}\left(\mathbf{y}_{1} ; \mathbf{a}\right)\right\|^{2}<\infty, n^{-1} \sum_{k=1}^{n}\left\|\dot{f}\left(\mathbf{y}_{k} ; \mathbf{a}\right)-\dot{f}\left(\widehat{\mathbf{y}}_{k} ; \mathbf{a}\right)\right\|=$ $o_{p}(1),\|\dot{f}(\mathbf{y} ; \mathbf{t})-\dot{f}(\mathbf{y} ; \mathbf{a})\| \leqslant H(\mathbf{y}, \mathbf{a})\|\mathbf{t}-\mathbf{a}\|$ при $\mathbf{t}$ из некоторой окрестности точки $\mathbf{a}$, $\mathrm{E} H\left(\mathbf{y}_{1}, \mathbf{a}\right)<\infty, \sup _{k \in \mathbb{N}} \mathrm{E} H\left(\widehat{\mathbf{y}}_{k}, \mathbf{a}\right)<\infty$, где $\|\cdot\|$ - евклидова норма.

Пусть $Y\left(\mathbf{y}_{k} ; \gamma\right):=\sum_{m=0}^{\infty} \gamma^{m} y_{k-m}^{2}, \sigma^{2}\left(\mathbf{y}_{k} ; \mathbf{a}\right):=\alpha /(1-\gamma)+\beta Y\left(\mathbf{y}_{k} ; \gamma\right), Z\left(\mathbf{y}_{k} ; \mathbf{a}\right):=$ $\sum_{m=0}^{\infty} \gamma^{m} \sigma^{2}\left(\mathbf{y}_{k-m-1} ; \mathbf{a}\right)$, все ряды сходятся в $\mathbf{L}_{1}$. Введем векторную функцию

$$
\mathbf{e}(\mathbf{y} ; \mathbf{a}):=\left(\frac{1}{(1-\gamma) \sigma^{2}(\mathbf{y} ; \mathbf{a})}, \frac{Y(\mathbf{y}, \gamma)}{\sigma^{2}(\mathbf{y} ; \mathbf{a})}, \frac{Z(\mathbf{y}, \mathbf{a})}{\sigma^{2}(\mathbf{y} ; \mathbf{a})}\right)
$$

Сформулируем результат об асимптотической равномерной линейности остаточного эмпирического процесса. Пусть $\langle\cdot, \cdot\rangle$ - евклидово скалярное произведение. 
Теорема 1. Если выполнены условия (i)-(iv), то при $0 \leqslant T<\infty u n \rightarrow \infty$

$$
\begin{aligned}
\sup _{x \in \mathbb{R},\|\mathbf{t}\| \leqslant T} \mid & U_{n}\left(x, \mathbf{a}+n^{-1 / 2} \mathbf{t}\right)-U_{n}(x) \\
& -2^{-1} x g(x)\left\langle\mathrm{E}\left[f\left(\mathbf{y}_{1} ; \mathbf{a}\right) \mathbf{e}\left(\mathbf{y}_{1} ; \mathbf{a}\right)\right], \mathbf{t}\right\rangle-G(x)\left\langle\mathrm{E} \dot{f}\left(\mathbf{y}_{1} ; \mathbf{a}\right), \mathbf{t}\right\rangle \mid=o_{p}(1) .
\end{aligned}
$$

Заметим, что при $\alpha=1, \gamma=0$ утверждение теоремы 1 совпадает с теоремой 3.1 из [3] об остаточном эмпирическом процессе в АRCH-модели, а при $f(\mathbf{y} ; \mathbf{t}) \equiv 1$ получаем теорему из [4] об остаточной эмпирической функции распределения.

3. Зная функцию $G(x)$, можно получить асимптотически эквивалентную ОМП оценку вектора а из системы уравнений $\sum_{k=1}^{n} \mathbf{e}\left(\widehat{\mathbf{y}}_{k-1} ; \mathbf{t}\right) L_{g}\left(\varepsilon_{k}(\mathbf{t})\right)=0$, где $L_{g}(x)=1+x \frac{g^{\prime}(x)}{g(x)}$. Поэтому естественно рассмотреть оценки, определяемые системой уравнений:

$$
\sum_{k=1}^{n} \mathbf{f}\left(\widehat{\mathbf{y}}_{k-1} ; \mathbf{t}\right) \psi\left(\varepsilon_{k}(\mathbf{t})\right)=0
$$

где $\mathbf{f}=\left(f_{1}, f_{2}, f_{3}\right)$, а $f_{i}$ и $\psi$ заданы априорно.

Определим матрицы $\mathbf{A}_{\mathbf{f}}=\left(a_{i, j}\right), \mathbf{R}_{\mathbf{f}}=\left(r_{i, j}\right)$, где

$$
a_{i, j}=\mathrm{E}\left[e_{i}\left(\mathbf{y}_{1} ; \mathbf{a}\right) f_{j}\left(\mathbf{y}_{1} ; \mathbf{a}\right)\right], \quad r_{i, j}=\mathrm{E}\left[f_{i}\left(\mathbf{y}_{1} ; \mathbf{a}\right) f_{j}\left(\mathbf{y}_{1} ; \mathbf{a}\right)\right],
$$

при этом $e_{i}(\mathbf{y} ; \mathbf{a})$ - компоненты вектора $\mathbf{e}(\mathbf{y} ; \mathbf{a})$. Теорема 1 влечет существование $n^{1 / 2}$-состоятельного решения (2) при обычных предположениях относительно $\psi$, см. [3]:

(v) $\psi$-непрерьвная функция, $\left.\operatorname{var}\right|_{-\infty} ^{\infty}[\psi]<\infty, \mathrm{E} \psi\left(\varepsilon_{1}\right)=0, J_{\psi}:=2^{-1} \int_{-\infty}^{\infty} x g(x) d \psi(x) \neq 0$.

ТЕорема 2. Пусть выполнены условия (i), (ii), функции $f_{i}$ удовлетворяют условиям (iii), (iv), а $\psi$ - условию (v), $\mathbf{A}_{\mathbf{f}}, \mathbf{R}_{\mathbf{f}}$ - невырожденные матрицы. Тогда с вероятностью, стремящейся $к$ единице, существует $n^{1 / 2}$-состоятельное решение (2), и для любого такого решения $\widehat{\mathbf{a}}_{n}$

$$
n^{1 / 2}\left(\widehat{\mathbf{a}}_{n}-\mathbf{a}\right) \stackrel{d}{\longrightarrow} N\left(\mathbf{0}, \mathbf{D}_{\mathbf{f}, \psi}\right), \quad \text { } \partial e \quad \mathbf{D}_{\mathbf{f}, \psi}=J_{\psi}^{-2} \mathrm{E}\left[\psi^{2}\left(\varepsilon_{1}\right)\right]\left(\mathbf{A}_{\mathbf{f}}{ }^{-1}\right)^{T} \mathbf{R}_{\mathbf{f}} \mathbf{A}_{\mathbf{f}}{ }^{-1} .
$$

K сожалению, функцию $\psi$, удовлетворяющую условию (v), удается построить только при известной $G(x)$. Поэтому, в действительности, теорема 2 описьвает параметрическую ситуацию.

Можно показать существование функций $\mathbf{f}$ и $\psi$, удовлетворяющих условиям теоремы 2 , таких, что соответствующие оценки имеют конечную чувствительность к грубым выбросам.

Автор выражает признательность М. В. Болдину за постановку задачи и ее обсуждение.

\section{СПИСОК ЛИТЕРАТУРЫ}

[1] S. W. Lee, B. E. Hansen // Econometric Theory. 1994. V. 10. P. 29-52. [2] R. D. Martin, V. J. Yohai // Ann. Statist. 1986. V. 14. P. 781-818. [3] M. V. Boldin // Math. Methods Statist. 2000. V. 9. № 1. Р. 65-89. [4] А. Е. Вязилов // УМН. 1999. Т. 54. № 4. С. 163-164. 